\title{
Erratum
}

Curr Genet (1992) 22: 407-413

\section{Genomic organization of a cellulase gene family in Phanerochaete chrysosporium}

\author{
Sarah F. Covert ${ }^{1 *}$, Jennifer Bolduc ${ }^{2}$, and Dan Cullen ${ }^{2}$ \\ 1 Department of Bacteriology, University of Wisconsin-Madison, Madison, WI 53706, USA \\ 2 Institute for Microbial and Biochemical Technology, Forest Products Laboratory, Forest Service, U.S. Department \\ of Agriculture, Madison, WI 53705-2398, USA
}

Received March 2/May 3, 1992

On page 409 of the paper starting on page 407 of Volume 22 there were unfortunately several errors in Fig. 2 of the following article. The figure is reprinted below.

GATCACACCCTGGGGGTACGTTGATGAGCTTGGCATCAGGAGCCTTCGTC TATTTCCTCCTTCGGGCCCGTGGGGCAGACGGTTCTCATATTCTCGGAAG CCTCTTTCAACGACGGTCAGCACGTTTGCCTGGCTTGCGCGCGACGAGCC GTTTCTCACTGTCAGGTTTACCCGCAGAAACGCAGGTGCGCGCTGCGCCT CGTCGGCAGCCTGCACGAGGCGAGCACGGTCATGAGCACCGTGGATAAAC ATTCCATTGGAGTACGAAGCTGCGCCCGGCCGCCTCGAAATTGTCGGGGC AAGTACTCGGTGCAGCCGCTGCAATTGTCCGTCTTGGCTCATGGGACCAC CTTCATCGCGACGGCGAGACACAGAGTAAAGCCGTGATGGTCGAACAGAC CGCTTCAGGGAGTGAGTATAAAGGCTGCATCCACAGCGCTGCTAATGACC AGGTCGCTCCAAGCATCCTTCACAAGGTTACCCGGTCTACTGTACGCACT TCTACTCAGAGTTGGAATCTAGAACTGACGACTTGCCTTGCAGTTGTCCC CATGTTCCGCGCCGCCGCACTCCTCGCTTTCACTTGTCTCGCCATGGTGT

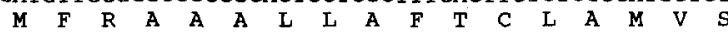
CTGGCCAACAGGCTGGCACCAACACGGCGGAGAACCACCCCCAGCTCCAG

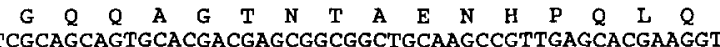

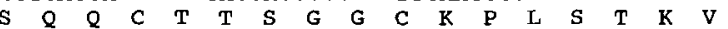
CGTCCTCGACTCGAACTGGCGCTGGGTCCACAGCACCTCGGGCTACACCA

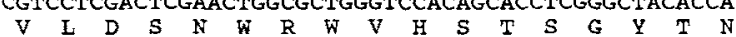
ACTGCTACACCGGCAACGAGTGGGAC...... (231)..... TGCGGTC

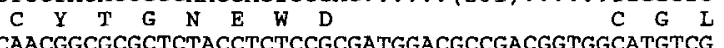
TCAACGGCGCGCTCTACCTCTCCGCGATGGACCCCGA G G AAGTACCCCGGAAACAAGGCTGGTGCCAAGTACGGAACTGGTTACTGCGA AAGTACCCCGGAAACAAGGCTGGTGCCAAGTACGGAACTGGT TACTGCGA CTCGCAGTGCCCGAAGGACATCAAGTTCATTAACGGCGAGgtgagctctc $\begin{array}{llllllllllllllllll}S & Q & C & P & K & D & I & K & F & I & N & G & E\end{array}$ actataccacgacgagaagtcacgagctgacaccgttctgctagGCTAAT GTCGGCAACTGGACCGAGACCGGCAGCAACACCGGTACGGGCAGCTACG $\begin{array}{lllllllllllllllll}V & G & N & W & T & E & T & G & S & N & T & G & T & G & S & Y & G\end{array}$ TACCTGCTGCAGCGAGATGGACATATGGGAGGCCAACAACGATGCCGCTG

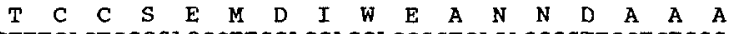
CTTTCACTCCCCACCCTTGCACCACCACCGGTCAGACCCGTTGCTCTGGG $\begin{array}{llllllllllllllll}F & T & P & H & P & C & T & T & T & G & Q & T & R & C & S & G\end{array}$ D A $R$ T T CAACTCGTTCCGCATGGGTGACAAGACCTTCCTCGGCAAGGGGATGACCG

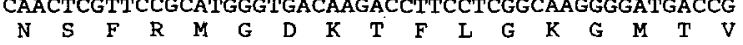
TCGACACCTCCAAGCCCTTCACCGTCGTCACCCAGTTCCTGACCAACGAC $\begin{array}{lllllllllllllllll}D & T & S & K & \text { P } & F & T & \text { V } & \text { V } & \text { T } & Q & F & \text { L } & T & N & \text { D }\end{array}$ AACACCTCCACCGGCACGCTCTCTGAGATC 1330

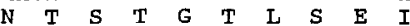

GATCTTCTTCTACGCCTGCTGCAGCTCACGGGGCCATGAATTTCTAGTGC TGTAGTGCTCGCGCGAGGCCTTCTATCCTCATCTGTGTCGTCAAGAGCCT CCAAATTGTCGGCCCAAAATCGCCAGGGTGAAGTCGCTGTTTATCCGCTG A ATCG TGCGCTGTCCACGAGEATGTCGCAGCGGGATGTCTCTGACATG GGGGTAGATGTGCGGAGAATTGGCAAAGGTATAAAGGCTCTGGGTCGCCG AGGGTTACAGCTCAGAGCACCAGTACTCT CTTGCAAAGCAGCTCCCCCAG AGCAACGGTCTCCCGGGTAAGTGTGTATTCGAGTGTAGCTATTACAATAC TGATTTGGTCTCCAGCAAAATGTTCCGCGCTTCAGCACTCCTCGCGTTCT

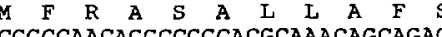
CTTTCGCTGCCATCGCGTACGGCCAACA

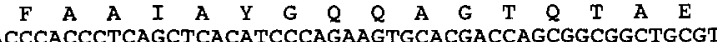
ACCCACCCTCAGCTCACATCCCAGAAGTGCACGACCAGCGGCGGCTGCGT CACCCAGGACACGACCGTCGTGCTCGACTCCAACTGGCGCTGGCTGCACT $\begin{array}{lllllllllllllllll}T & Q & D & T & T & V & V & L & D & \text { S } & \text { N } & \text { W } & R & \text { W } & L & \text { H } & S\end{array}$ $\begin{array}{llllllllllllllll}V & Q & G & Y & T & N & C & Y & T & G & N & E & W & D\end{array}$ (273) ...... GACGGTGGCATGTCCAAGTTCCCCAACAACAAGGCTGGT $D G G \quad M \quad S \quad K \quad F \quad P$ N $N$ K A G GCCAAGTACGGCACTGGCTACTGCGACTCGCAGTGCCCGCGGGACATCAA

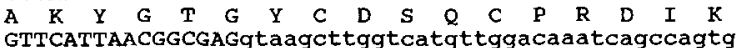
$\underset{F}{\text { GTTCATTAACGGCGAGgtaagcttggtcatg ttggacaaatcagccagtg }}$
ctcatctcgcgettccogtagGCGAACGTCGAGGGCTGGAACGCCACCAG A $N$ V E G W N A T $S$ CGCGAACGCGGGTACTGGTAACTACGGCACCTGCTGCAGCGAGATGGATA $\begin{array}{lllllllllllllllll}\text { A } & \text { N } & A & G & T & G & N & Y & G & T & C & C & S & E & M & D & I\end{array}$ TCTGGGAGGCGAACAACGATGCTGCTGCCTTCACTCCCCACCCCTGCACC

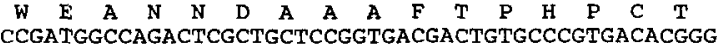
$\begin{array}{lllllllllllllllll}T & D & G & Q & T & R & C & S & G & D & D & C & A & R & D & T & G\end{array}$

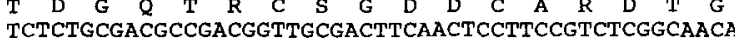

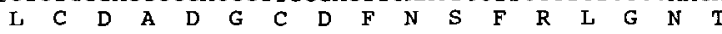
CGACGTTCCTCGGCAAGGGCATGACCGTCGACACCAGCAAGCCCTTCACC $\begin{array}{llllllllllllllll}T & F & L & G & K & G & M & T & V & D & T & S & K & P & F & T\end{array}$ GTCGTCACGCAGTTCCTGACGAACGACAATACCTCGACGGGCACGCTCAG $\begin{array}{lllllllllllllllll}V & V & T & Q & F & L & T & N & D & N & T & S & T & G & T & L & S\end{array}$ CGAGATC 1107

GCTGGAGGATGTGAGTCGGTGAAAGGCAGAGCTCCACCGCCCTCCACCAG CGATCCAGCTCTACGTGAGTCGGACTTCCTCTGAACGGAGCAGAACTGCG AGGAACT GACGCGTCGCGACAGACACAAAAGGCCTCACTCGGGTCTCCCA GGTCAACAAACGGTCACCGGTCAAAAACTTCACTGGTGAGTCCGCCATTG TGCATGGCTGTCGGTCGACGTGCCGCTAACCGAGTTGGACGCACACTGGC AGACGGTTGCAAAAATGTTCCGCAAGGTCGCACTCATCGCATTCACGCTG $\begin{array}{llllllllllll}M & F & R & K & V & A & L & I & A & F & T & L\end{array}$

GCCGCGATGGCCGTCGGCCAGCAGGTCGGCACGAACACGGCCGAGAACCA

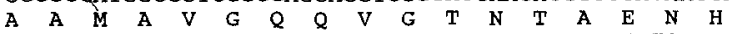
CCCGACGCTCACGTCGCAGAAGTGCACGACCGCGGGCGGCTGCGTGAGCC $\begin{array}{lllllllllllllllll}P & T & L & T & S & Q & K & C & T & T & A & G & G & C & V & S & Q\end{array}$ U I V I D A TCGGGCTACACCAACTGCTACACTGGCAACGAGTGGGACGCGACGCTCTG

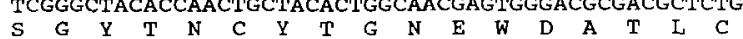
CCCCGACGGCGCGACGTGCGCGGCCAACTGCGCGCTCGACGGC . . . . $\begin{array}{llllllllllllll}P & D & G & A & T & C & A & A & N & C & A & L & D & G\end{array}$ (255) . . . . GGCGCCAAGTACGGCACTGGCTACTGCGACTCGCAGTGCC $G \quad A \quad K \quad Y \quad G \quad T \quad G \quad Y \quad C \quad D \quad S \quad Q \quad C$ CGAAGGACATCAAGTTCATCAACGGCGAGgtgcgtgcaggcgcgatcgtt $K$ D I $K$ F I N G E cgattgggtgttatccccccttgctgactcgtccttccccogcacatcco cagGCAAACGTCGAGGGATGGACTGGCACCAGCGCCAACGCAGGCACCGG

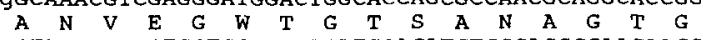
CACCTACGGCACCTGCTGCAACGAGATGGACATCTGGGAGGCGAACAACG

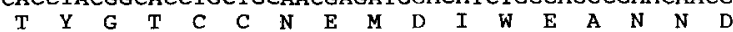
ACGCCGCGGCGTTCACCCCGCACCCGTGCACGACCACCGGCCAGACGCGC $\begin{array}{llllllllllllllll}\mathbf{A} & \mathbf{A} & \mathbf{A} & \mathbf{F} & \mathrm{T} & \mathbf{P} & \mathbf{H} & \mathbf{P} & \mathbf{C} & \mathbf{T} & \mathbf{T} & \mathbf{T} & \mathrm{G} & \mathbf{Q} & \mathbf{T} & \mathbf{R}\end{array}$ TGCTCGGGCGACGACTGCGCCCGGGACACCGGCCTGTGCGACGCCGACGG

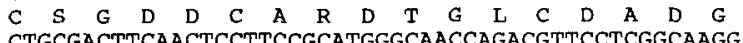

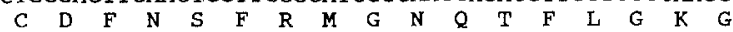
GCCTCACCGTTGACACCAGCAAGCCGTTCACGGTCGTCACCCAGTTCCTG $\begin{array}{llllllllllllllll}L & T & V & D & T & S & K & P & F & T & V & V & T & Q & F & L\end{array}$ ACGAACGACAACACGACGACGgGCACGCTCAGCGAGATC 1039

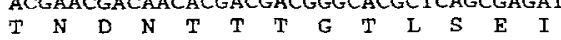

Fig. 2. Partial nucleotide and deduced amino-acid sequences of representatives of cosmid Groups $2 b$ (upper), 3 (middle), and 4 (lower). Dots represent regions which have not been sequenced, and numbers in parenthesis indicate estimated distance in nucleotides between the two segments. Introns are in lower case letters. Putative promoter elements are underlined. Nucleotides 1067-1116, 844-933, and $776-825$ of Groups $2 \mathrm{~b}, 3$ and 4, respectively, encode 17 amino acids which are indentical to the CBHI peptide sequence of Uzcategui et al. (1991). EMBL accession numbers are Z11733/Z11726 (2b); Z11730/Z11727 (3); and Z11731/Z11729 (4)
800

850

900

950

1000

1050

1100

50

150

200

300

350

400

450

500

550

600

650

700

800

850

900

950

1000 